\title{
Modeling a drum motor for illustrating wearout phenomena
}

\author{
Olaf Enge-Rosenblatt ${ }^{1}$, Christian Bayer ${ }^{1}$, Joachim Schnüttgen ${ }^{2}$ \\ ${ }^{1}$ Fraunhofer Institute for Integrated Circuits, Division Design Automation, \\ Zeunerstraße 38, 01069 Dresden, Germany \\ \{Christian.Bayer; Olaf.Enge\}@eas.iis.fraunhofer.de \\ ${ }^{2}$ Interroll Holding $\mathrm{GmbH}$, Interroll Research Center, \\ Lothforster Straße 32-40, 41849 Wassenberg, Germany \\ J.Schnuettgen@interroll.com
}

\begin{abstract}
In this contribution, a model of a drum motor is presented. This model was designed for description of dynamic behaviour of the drum motor as well as for the possible implementation of several wearing phenomena. Using this model, a better understanding of wear and tear phenomena has been achieved by carrying out a considerable number of simulation runs using different operational and wearing conditions. Using this information, important knowledge about detection of wearout signs was able to be gained.

Often, mathematical models with different levels of detail are used. In these cases, it may be a difficult task to obtain reliable parameters. In this paper, we present three different approaches for establishing a model structure and for the determination of needed parameters. This way, we were able to define every part of the model with an appropriate level of detail and equip them with adequate parameter values.
\end{abstract}

Keywords: drum motor; mathematical model; wearout phenomena modeling; parameter determination; condition monitoring

\section{Introduction}

Applications of mathematical models of technical systems are widespread in today's product development cycle. Mathematical models help to increase the understanding of physical properties of a product. The usage of mathematical models in the design phase allows investigations of functional properties under changing operational conditions. Both properties and operational conditions are described in the models by certain parameters. In the early phase of product development, only a certain range of values for these parameters is needed. Later on, these pa- rameters have to be determined with higher accuracy to benefit from the model-based investigations.

Correct and robust operation under changing circumstances is the most important requirement concerning machines and facilities in today's industry. Additionally, all equipment must guarantee a very high level of availability. These two demands are competing against each other because every technical system is characterized by a certain appearance of wear and tear. This applies to mechanical and electrical systems but also for any other physical domain. This appearance of wear and tear increasingly causes a less correct operation of any technical system with progressing time of operation. Therefore, compliance checks concerning the allowed tolerances have to be performed either in certain time intervals or depending on the current condition of wearing. However, those checks take time and, therefore, decrease the machine's availability.

Using a mathematical model of a machine or a facility that is able to reconstruct phenomena of wearing is one promising way of getting out of the dilemma. Still, the model must be able to describe functional and dynamic properties, too. That is the reason why such mathematical models cannot be implemented in an easy and straight forward manner. The model structure developed first has to be laid out with necessary parameters. Some of them can be calculated while other ones may only be measured. Calculation may be performed analytically or, e.g., by using a Finite Element model. Parameter measurements mostly need considerable effort for establishing an appropriate test set-up. All three methods were applied for the development of the model presented here. Using a well-parameterized model, we can carry out investigations about impacts of effects of wear and tear on functional properties of a machine or a facility. 


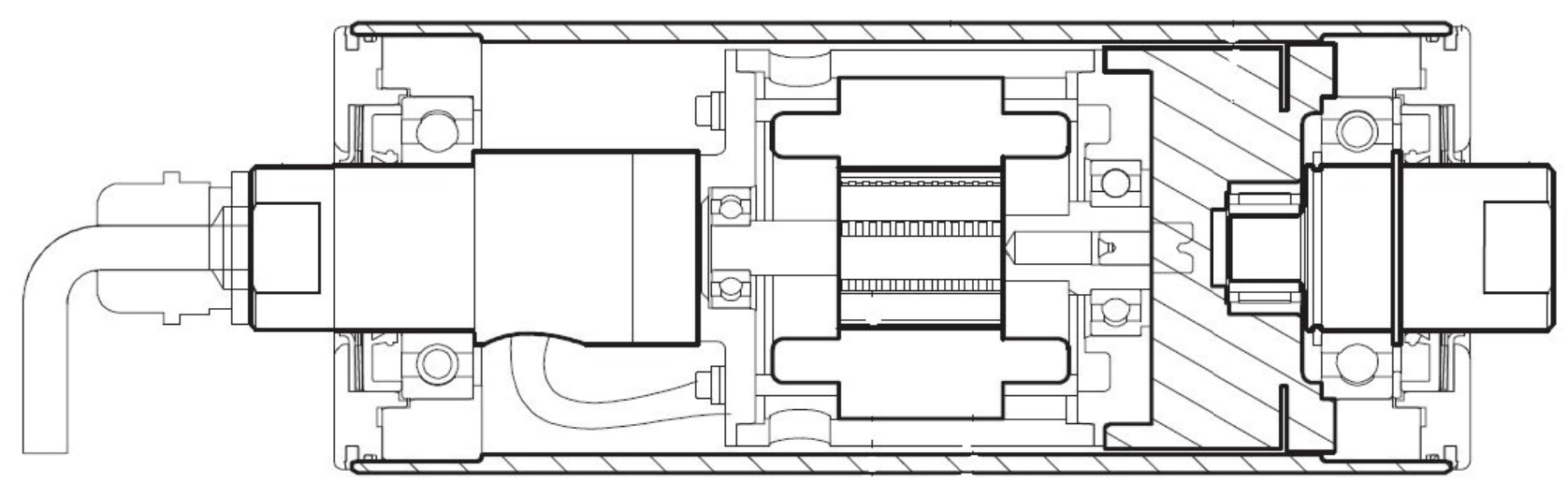

Figure 1 Sectional view of drum motor

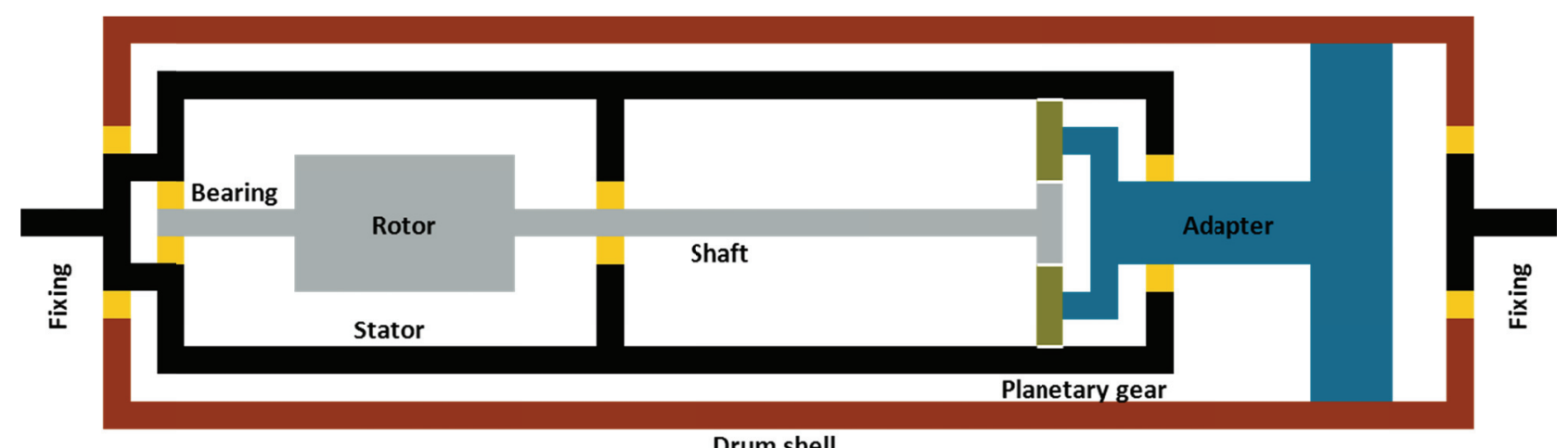

Drum shell

Figure 2 Sketch of the components of the drum motor

In this paper, a mathematical model of a drum motor is presented. This model regards important wearout phenomena occurring within the drum motor's components. Important model parameters are determined by analytical investigations, by Finite Element methods or by extensive measurements. Some parameters are included into the model by look-up tables. Finally, some simulation results performed to prove the functional properties of the model are given.

\section{Design of a drum motor}

A drum motor (or sometime referred to as a motorised pulley) is a component for driving a pulley for conveyor belts. A sectional view of such a motor is depicted in Figure 1. Figure 2 shows a sketch of the main components belonging to a typical drum motor. From both figures, one can understand the operation of the drum motor. The stator of the electric motor and the other black parts (including the ring wheel of the planetary gear) are fixed at the outer space. The grey part consists of the rotor of the electric motor and the shaft connected to the input of the planetary gear (sun wheel). Both are driven by the motor's electromagnetic force. The carrier of the planetary gear is shown in blue colour (Adapter) while the planetary wheels are filled light green. All yellow parts stand for roller bearings. The two outer bearings are the main bearings of the drum motor. They have to resist the forces caused by the belt and its load. The three inner bearings have to guide the rotor of the electric motor and the gear's carrier. Finally, the drum shell is depicted in red colour. It is tightly attached to the adapter and, therefore, driven by the planetary gear carrier.

This mechanical system was modeled using Modelica because this multi-physical language is well-prepared for implementation of different approaches for parameter definitions [4]. The Modelica model diagram is shown in Figure 3. The connection to a model of the electrical subsystem of the electric motor (including controlling algorithms and power inverter) is realised by one input signal (torque) and two output signals (angle and angular velocity). The electrical subsystem's model is provided by a third party ([5]) and will not be discussed here in detail. 


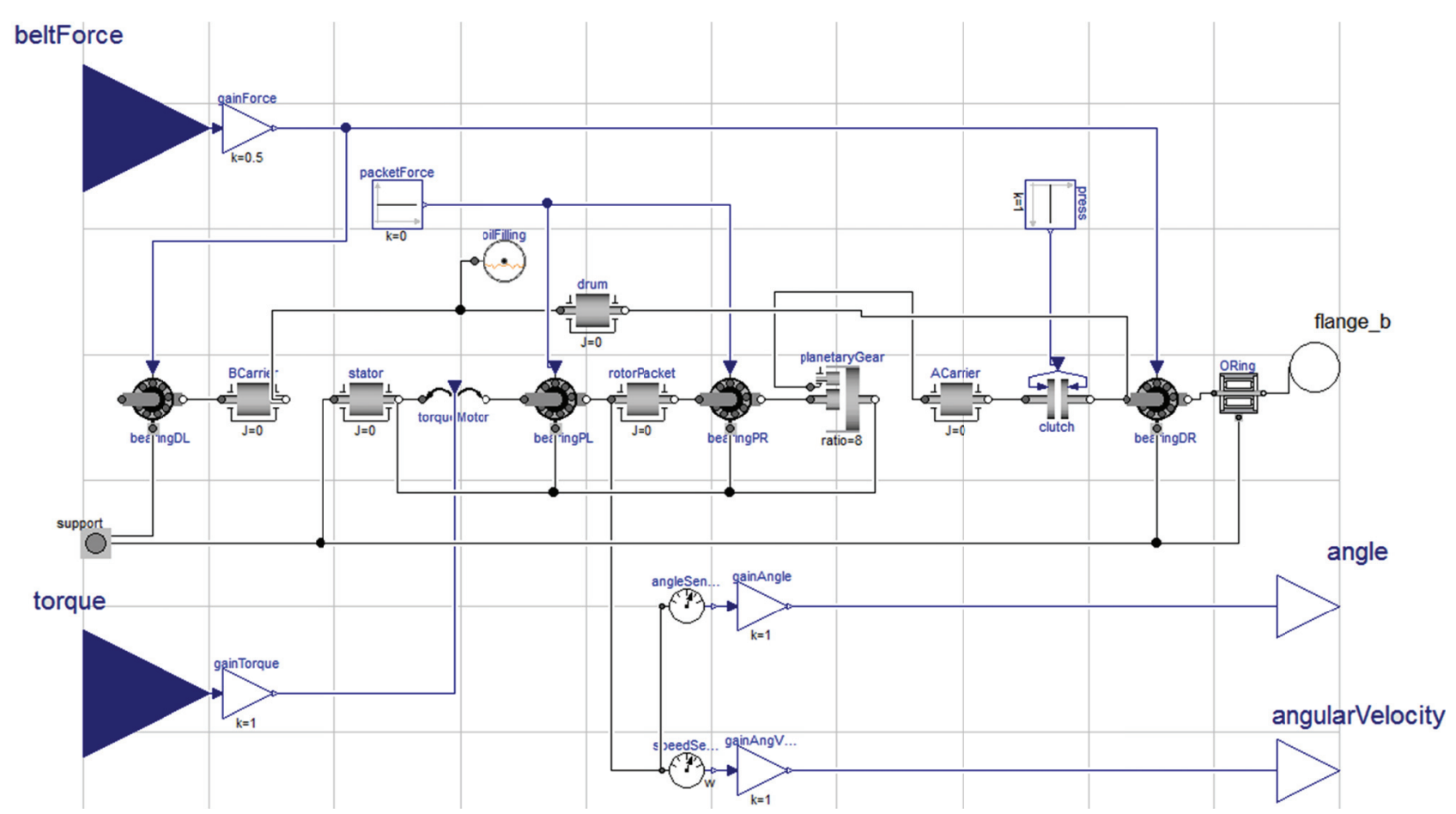

Figure 3 Modelica model diagram of the drum motor

\section{Modelica model}

The Modelica model (see Figure 3) consists of some main modules each describing a mechanical subsystem. First, there are the main roller bearings ("bearingDL" and "bearingDR") between the drum shell ("drum") and the fixed part ("support") as well as the smaller roller bearings ("bearingPL" and "bearingPR") between the rotor of the electric drive ("rotorPacket") and the fixed stator ("stator") or the planetary gear carrier ("ACarrier"), respectively. Second, the friction because of the O-rings is taken into account ("ORing") and, third, a gear is included into the model ("planetaryGear"). The additional modules (e.g. "oilFilling") are not of importance here.

All of the used sub-models can be supplemented by several wearout phenomena. The model's level of detail defines which phenomena can be included. The more details are implemented in a model the more phenomena can mathematically be described. Some effects can be formulated by investigating physical relations, other effects, however, have to be described by general behavioural models which only give an approximated representation of static and dynamic properties regardless of real physical reasons behind them.

In the following, we present the most important parts of our model of a drum motor.

\subsection{Roller bearings}

The model of the roller bearings is implemented as a cylindrical joint component with only one rotational degree of freedom. Hence, it is designed much simpler than e.g. in [1] or [11]. The model is realized as an extension of the BearingFriction model of the Modelica Standard Library [9]. That means that both rotational axes of inner and outer ring are identical. The friction definition of the Standard Library has been complemented by friction effects caused by the rolling elements (balls in many cases), which are placed between the inner and outer ring of the roller bearing (see e.g. [12]). The model is used for belt drive applications, where an external force can act on the bearing. Hence, the BearingFriction model was extended by a RealInput interface to connect any external force value applied to the bearing. Typically, the direction of this force is fixed and thus the force is absorbed by all balls located on one halfspace of the bearing.

Figure 4 shows a simple sketch of a roller bearing. It illustrates the application and the distribution of forces. The belt force $F_{B}$ is divided into several sub-forces $F_{B}^{i}$. The resulting additional friction torque depends on the mechanical strain of the balls and their slight deformation. 


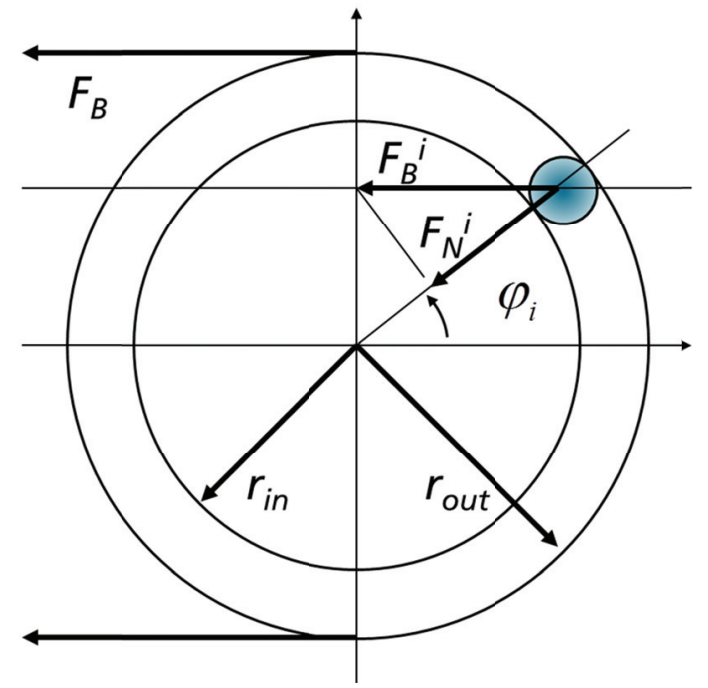

Figure 4 Sketch of a roller bearing

The pressure of each ball can be described by the Hertzian contact stress ([6], [7], [10]), which is basically modeled by a non-linear spring system. The displacement value for this model originates from a very small relative shift between the axes of inner and outer ring when a force is applied. Hence, the displacement value can be calculated analytically for each ball with respect to its angular position $\varphi_{i}$. We found that it is a good approximation to consider the displacement $\delta_{i}$ to be proportional to the term $\cos \varphi_{i}$. According to the Hertzian contact model the normal force to a ball then becomes

$$
F_{N}^{i}=\widetilde{F}_{B}\left(\cos \varphi_{i}\right)^{3 / 2},
$$

where $\widetilde{F}_{B}$ is a force value which will be determined by the external force applied to the bearing. Figure 4 shows that the normal force $F_{N}^{i}$ is a projection of the partial external force $F_{B}^{i}$ to the radial direction. $F_{B}^{i}$ can therefore be expressed as

$$
F_{B}^{i}=\frac{F_{N}^{i}}{\cos \varphi_{i}}=\widetilde{F}_{B} \sqrt{\cos \varphi_{i}} .
$$

From equation (2), the distribution of the external belt force according to angular ball positions is given. Note that only balls of the respective half-space are considered as the remaining balls are not affected by the force. Finally, the force value $\widetilde{F}_{B}$ is determined by the fact that all sub-forces $F_{B}^{i}$ sum up to the belt force $F_{B}$ and thus reads

$$
\widetilde{F}_{B}=\frac{F_{B}}{\sum_{i} \sqrt{\cos \varphi_{i}}},-\frac{\pi}{2} \leq \varphi_{i} \leq \frac{\pi}{2} .
$$

The force value $\widetilde{F}_{B}$ is actually time-dependent. The force acting on one ball changes not only with angular position but also with the number of balls present in the half-space. Eventually, this causes slight fluctuations of the torque needed to operate the bearing continuously.

One of the models purpose is to simulate abrasion, which can be treated as an increased rolling friction $F_{R}^{i}$. The friction force is proportional to the known normal force $F_{N}^{i}$ andl thus depends further on a factor $k_{R}^{i}$ for each ring, which might be a function of the angular position $\varphi_{i}$ measured relative to the ring under consideration. We defined these factors by two look-up tables for all angular positions between 0 and $2 \pi$. This introduces high flexibility for the definition of damaged spots on the rings. The absolute value of the friction torque due to rolling balls then reads

$$
\tau_{R}=\sum_{i} F_{N}^{i}\left(r_{\text {in }} k_{R, \text { in }}^{i}+r_{\text {out }} k_{R, \text { out }}^{i}\right) .
$$

where $r_{\text {in }}$ and $r_{\text {out }}$ are the radii of inner and outer ring. For abrasion simulation it is necessary to calculate the angular ball positions relatively to the ring positions, which are known from the connecting flanges. Moreover, the rotation speed of the balls differs from the speed of inner and outer ring and the rings might both rotate. However, they are a suitable reference system and the relative positions become

$$
\begin{gathered}
\varphi_{i}^{\text {in }}=-\frac{\varphi_{\text {in }}-\varphi_{\text {out }}}{1+\frac{r_{\text {in }}}{r_{\text {out }}}}+\varphi_{i, 0} \\
\varphi_{i}^{\text {out }}=\frac{\varphi_{\text {in }}-\varphi_{\text {out }}}{1+\frac{r_{\text {out }}}{r_{\text {in }}}}+\varphi_{i, 0} .
\end{gathered}
$$

Equations (5) describe the angular position of a ball with respect to the inner and outer ring, respectively, where $\varphi_{i, 0}$ denotes an initial position of the ball. Using (5), we can implement wearout phenomena depending on relative angles between rolling elements and both rings. This way, the most important forms of damage of roller bearings (e.g. dull surface, surface depressions like pittings) can be modeled by a variable friction depending on momentary angles of inner and outer ring. 


\subsection{O-ring friction}

The drum motor is partly filled with oil which must not leak out during operation. At least two so-called O-rings are used for sealing both ends of the drum shell preventing the oil from leaking out. But they introduce high friction values and, therefore, they are of very high influence on dynamic behaviour of the drum motor.

To model such friction, extensive measurements were carried out by Interroll as will be described in Section 4. The goal of these investigations was not only to account for the non-linear behaviour but also to obtain useful absolute values for the friction torque. We found that the friction values depend mainly on the material of the O-rings as well as on the used lubricant (used or used not, lubricant grade, lubricant amount). But the friction behaviour for a fixed combination of material and lubricant shows a simpler structure in comparison with the friction behaviour of roller bearings. A first approximation of the friction may be a combination of a velocityproportional share and a nearly constant share. Hence, it can be written like

$$
\tau_{O}=k_{O} \omega+\tau_{O, 0},
$$

where $\omega$ is the rotational speed of the drum shell. The parameters $k_{O}$ and $\tau_{O, 0}$ have to be found by performing appropriate experiments. A second approximation was implemented by full correlation of torque with rotational speed by using a look-up table of measured values. To this end, the standard library model BearingFriction was finally used to model the O-rings because it offers such possibilities.

For both approximations, we assumed that O-ring friction does not vary with any outer load. The belt forces and any load in axial direction are completely absorbed by the main bearings. Hence, wearout phenomena at O-rings are expected to be uniform, i.e. they are not dependent on the drum shell's angle. But we found that O-ring friction vary both with hours of operation - because of wearing in effects - and with increasing wearout, e.g. by abrasion. These influences have to be determined by extensive measurements, too.

\subsection{One-stage gear}

The drum motor includes a single-stage or multistage planetary gear, which can be implemented by the standard library model IdealPlanetary or a combination of two of it. However, it does not include any features to emulate wearout effects. As a first approach the usually existing backlash and rotational stiffness can be inserted between the driving shaft and the gear's input wheel by a rotational spring damper system. The backlash influences the dynamic behaviour of the drum motor and is a measure for abrasion.

Gears have special kinds of tooth flanks to minimize abrasion [2], [13]. Most gears use the evolvent tooth flank, which ensures that there is no grinding between teeth and that the transmitted torque has no ripple. During operation the flank might degrade due to high loads or improper maintenance. Even teeth may break off under certain circumstances. As it would be very challenging to model these effects through multi-body simulation, we developed a Finite Element (FE) model starting from a two-wheel gear. In the ideal case, both wheels change their angular position simultaneously but with opposite direction. Actually, the driven wheel moves first and yields a slight deformation of teeth according to the Hertzian contact model. This effect is shown in Figure 5 for one wheel. The torque applied to the driven wheel is transmitted by this means. The deformation leads to a little deviation $\Delta \varphi$ in angular position of both wheels and depends also on the absolute position $\varphi$ of the wheels, as the contact point moves along the evolvent. The transmitted torque thus is a function of $\Delta \varphi$ and $\varphi$ which is determined by the FE simulation.

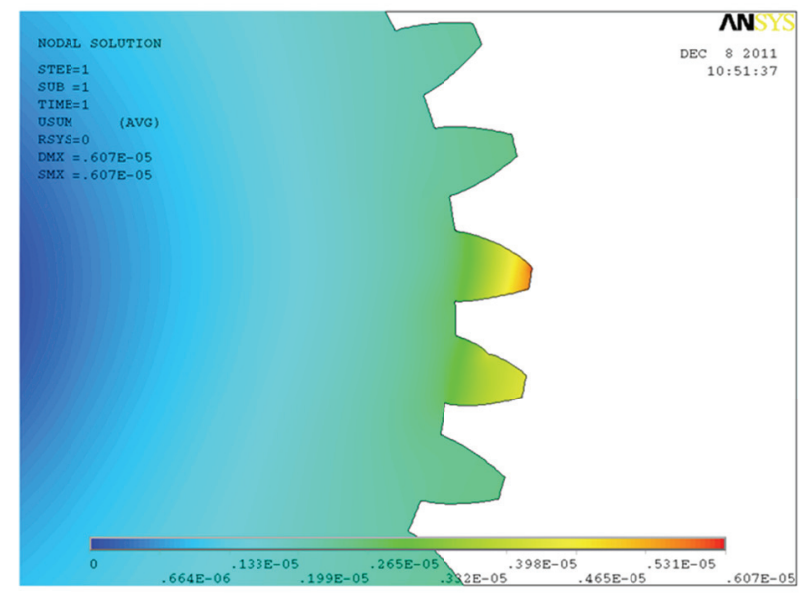

Figure 5 Gearwheel with stretching of teeth (Note that the deformation is displayed using a magnification of 100 in relation to the shape of the wheel)

Figure 6 shows the resulting torque function for two gear wheels of equal size. We can deduce that for small loads, i.e. for small $\Delta \varphi$, the transmission is independent of the angular position $\varphi$ and depends linearly on $\Delta \varphi$. However, for heavy loads the 
transmission function becomes non-linear and also depends on $\varphi$. Note that the torque function is periodic in $\varphi$.

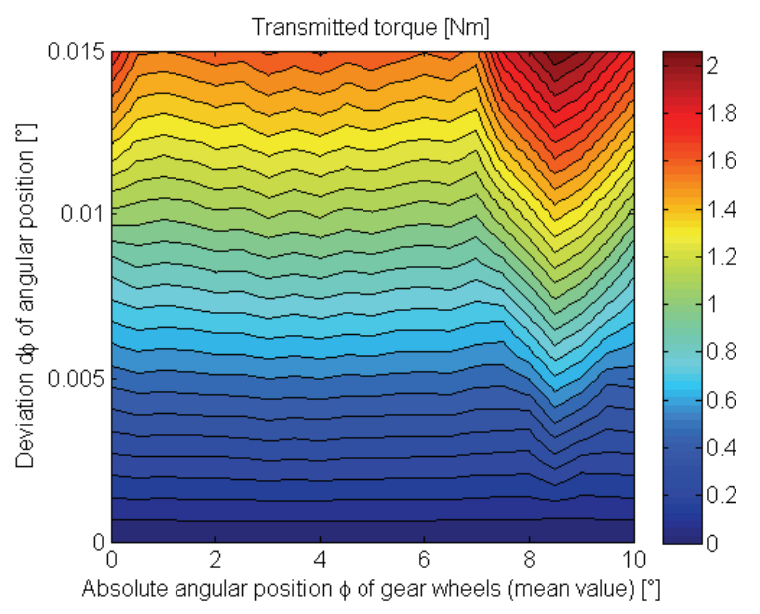

Figure 6 Dependency of torque on both angles and their difference

The results of the FE simulation can be used to develop a Modelica model of any gear wheel combination. The investigation of different tooth flanks and their abrasion is thus possible and even the effect of missing teeth can be evaluated.

\subsection{Environment of the model}

The drum motor is intended for belt drive systems. Such environments can be modeled by the commercial Belts library, which includes elements for the static and dynamic analysis of belt drive systems. The key components are belt spans and pulleys. We added interfaces to our drum motor model such that it can act as a pulley and is fully compatible to the library components. This way we are able to simulate the motor within a realistic environment and to investigate the effects of different signs of wear and tear.

Our Modelica model does not include the electric motor. This component was provided by a third party as a Matlab Simulink model [5]. It provides the driving torque and uses angular position and velocity from our model to control the electric motor. The cosimulation was carried out using the Dymola block feature in Simulink (see [8]). The purpose of this combined model is to investigate the influence of mechanical properties and setups to the electronic parts and controller of the motor. But these investigations are not content of this paper.

\section{Determination of parameters}

Determination of parameters used within the model is a very important task. Some parameters can be read from geometrical values like masses and moments of inertia. But other parameters like friction coefficients need to be determined by specific measurements carried out using real devices.

For an appropriate assignment of parameters needed to measure, a number of roll-out tests were performed. These tests were focused on the determination of friction losses because of bearings and $\mathrm{O}-$ rings without any influence from electric motor or gear. To this end, a single return pulley was accelerated to a certain rotational speed. After that, the pulley is retarded only by friction torques in bearings (first test set-up) or by friction torques in bearings and due to friction between drum shell and O-rings (second test set-up).

Temperature and lubrication are additional physical quantities influencing the friction torques. But temperature can be ignored if all measurements are carried out within a range of temperature like in a typical use case. Lubrication, if effected correctly, was found to be of no noticeable influence.

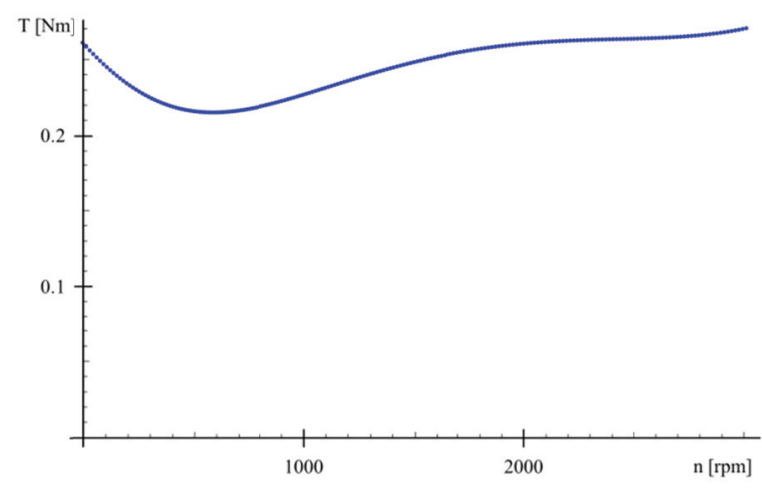

Figure 7 Friction torque without any O-ring

Figure 7 shows the curve of friction torque measured across rotational speed if no O-rings are attached to the test set-up. It is a result of retarding friction because of bearings only. The curve shows a shape which is nearly independent on rotational speed because torque values range from 0.2 to $0.3 \mathrm{Nm}$ (see also [12]). In a first approximation the friction torque is, therefore, implemented in the model by a constant value of Coulomb friction. A better integration of the measured curve can be performed using a look-up table containing momentary friction torque values depending on rotational speed.

Figure 8 depicts the measurement result if both outer O-rings are attached to the drum shell. This is a 
result of retarding friction because of bearings and O-rings. The curve shows a non-linear dependency on rotational speed. For simplification, the curve is split into one part with constant friction (approximately $0.95 \mathrm{Nm}$ ) and one part with a constant coefficient for friction depending linearly on rotational speed. The constant value is about $0.1 \mathrm{Nm} / 1000 \mathrm{rpm}$. Here again, a better integration of the measured curve can be performed via a look-up table.

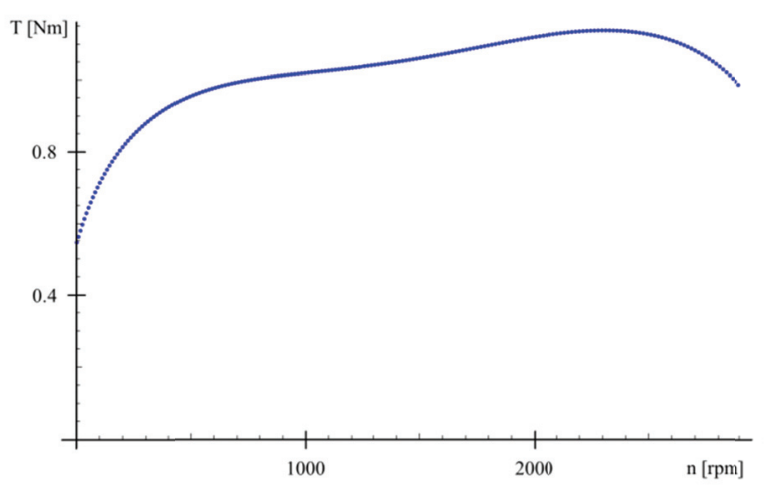

Figure 8 Friction torque with O-rings

\section{Simulation results}

As an example, we present simulation results regarding the roller bearings. Because of lack of measurement results up to now, we can unfortunately only present simulation results. These results were obtained using Dymola [3]. The application environment of the drum motor consists of a load by a constant transversal force (like force $F_{B}$ in Figure 4). This force is induced by the belt and reaches up to $1 \mathrm{kN}$ in a typical application.

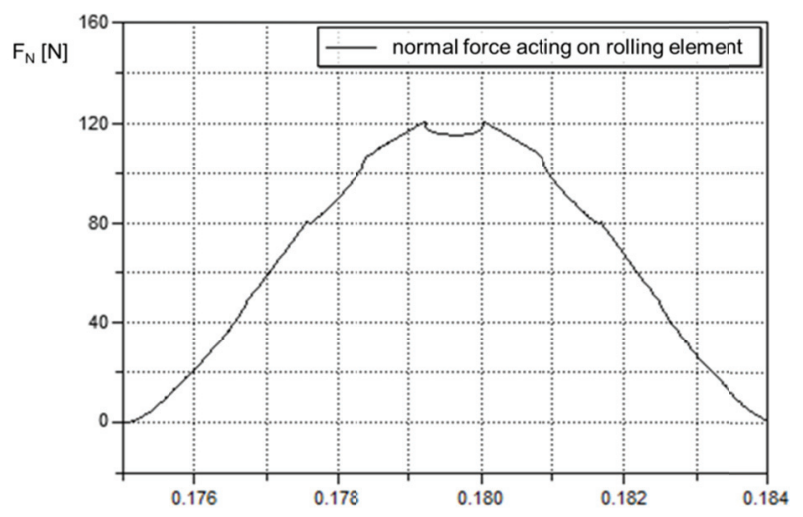

Figure 9 Normal force against time acting on one ball located within the force loaded halfspace of the roller bearing
First we investigated the normal force acting on the balls of a bearing. This gives an impression how the bearing model works and where the torque ripples originates from. Figure 9 shows the result across time for the motion of one ball within the forceloaded half space of the bearing (which is located in the half circle on the right hand side in Figure 4). The normal force is expected to be zero within the other half space (left hand side in Figure 4). Furthermore, we assume that there are no slip effects between ball and inner or outer ring of the bearing. Hence, the same curve shape results when depicting the normal force across the angular position of the ball instead of time. The friction torque for the one considered ball can then be determined from the normal force like shown in Section 3.1. This way, the ball-specific friction value can be calculated at every point in time depending on the ball's position and the bearing's transversal load force. Applying this result for every ball of the bearing, the complete friction can be calculated from present bearing angle and the load force.

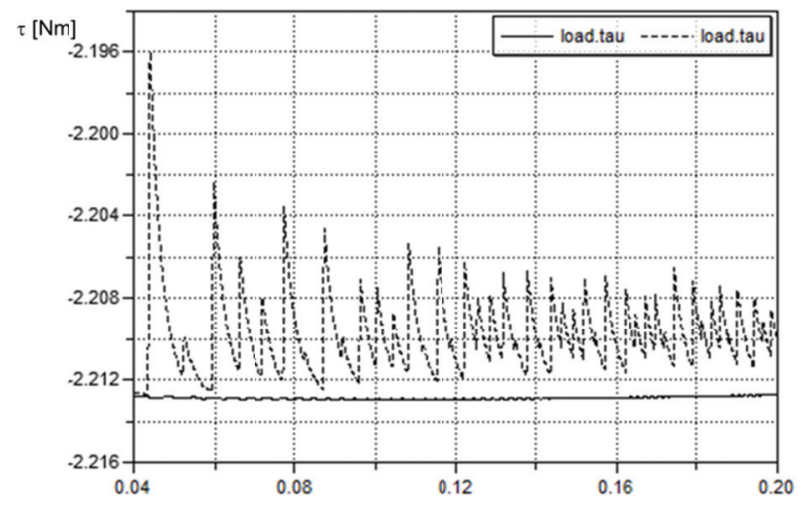

Figure 10 Torque transmitted to the load displayed across time without damage (solid line) and with localized damage on the outer ring (dashed line)

To investigate the resulting torque ripple at a rotating load (not to be mistaken for the transversal load force), the drum motor was driven by a constant torque and the rotational load was emulated by a linear and velocity dependent damper. To show the effect of a spot damage at one of the rings, such a damage was introduced by a localized increase of the friction coefficient $k_{R}$. This increase was calculated dynamically using the present bearing angle. We compared the torques acting on the load with and without spot damage, respectively. Figure 10 shows the results. Both torques are depicted across time for a certain range after the transient effect has been vanished. The torque without bearing damage (solid 
line) is nearly constant during the complete simulation interval. Contrastingly, the torque with bearing damage (dashed line) shows distinct ripples. The ripples' magnitude order is about $0.5 \%$ of the torque's mean value. That seems to be not so high but it is enough to detect some differences within a frequency plot. This way, a damage of sufficient dimension could be detected by a dedicated condition monitoring system.

\section{Conclusions}

We presented an approach to simulate wear and tear phenomena within complex systems. For the case of a drum motor we proposed three different methods of modeling and parametrising components thereof. The roller bearing was described analytically, whereas the gear was simulated using the Finite Element method. A third access to unknown parameters is measurement as shown with the O-rings. Using these well-parameterised models, we were able to establish a behavioural description for some important wearout effects with the drum motor. Hence, these models can be used to predict the behaviour of a worn system within its usual environment. This opens the possibility to investigate some consequences of wearout effects in several simulation runs in order to establish design rules for condition monitoring algorithms and thus support the development of adapted condition monitoring systems. This in turn allows for improved maintenance strategies and reduced costs.

\section{Ackknowledgement}

This work was funded by the German Federal Ministry of Economy within the project AutASS.

\section{References}

[1] Ashtekar, A.; Sadeghi, F.; Stacke, L.-E.: Surface defects effects on bearing dynamics. Proceedings of the Institution of Mechanical Engineers, Part J: Journal of Engineering Tribology, Vol. 224, No. 1, pp. 25-35, 2010

[2] Colbourne, J.R.: The geometric design of internal gear pairs, AGMA Technical Paper, 87 FTM 2, 1987

[3] Dymola 7.3, Dassault Systèmes
[4] Fritzson, P.: Principles of Object-Oriented Modeling and Simulation with Modelica 2.1, IEEE Press, 2004

[5] Herold, T.; Franck, D.; Lange, E.; Hameyer, K.: Extension of a d-q model of a permanent magnet excited synchronous machine by including saturation, cross-coupling and slotting effects. IEEE International Electric Machines Drives Conference (IEMDC), Niagara Falls, Ontario, Canada, 15-18 May, 2011, Proc. pp. 1363-1367

[6] Hertz, H.: Über die Berührung fester elastischer Körper. Journal für die reine und angewandte Mathematik, 92:156-171, 1881

[7] Hertz, H.: Über die Berührung fester elastischer Körper (On the contact of rigid elastic solids). In: Miscellaneous Papers. Jones and Schott, Editors, J. reine und angewandte Mathematik 92, Macmillan, London, pp. 156ff., 1896

[8] MATLAB® 2010a, The MathWorks, Inc.

[9] www.modelica.org/libraries/Modelica/

[10] Paland, E.G.: Technisches Taschenbuch. INA Schaeffer KG, 2002

[11] Stacke, L.-E.; Fritzson, D.: Dynamic behaviour of rolling bearings: simulations and experiments. Proceedings of the Institution of Mechanical Engineers, Part J: Journal of Engineering Tribology, Vol. 215, No. 6, pp. 499-508, 2001

[12] Tan, X.; Modafe, A.; Ghodssi, R.: Measurement and modeling of dynamic rolling friction in linear microball bearings. Journal of Dynamic Systems, Measurement and Control, Transactions of the ASME, 128 (4), pp. 891-898, 2006

[13] Zhuravlev, G.A.; Ageev, A.I.: Gear Teeth Bending Strength Analysis. Izvestia vyssih ucebnyh zavedenij. Masinostroenie (11), pp. 44-48, 1978 